\title{
Diseño de Ayuda Técnica para Terapia Física Enfocada a Personas con Paraplejia: Revisión de la Literatura
}

\author{
Design of a Technical Aid for Physical Therapy Focused on \\ People with Paraplegia: Literature Review \\ M. Miranda-Bañuelos ${ }^{1}$, E. G. Meraz-Tena' ${ }^{1}$, C. O. Balderrama-Armendáriz ${ }^{1}$ \\ ${ }^{1}$ Universidad Autónoma de Ciudad Juárez
}

\section{RESUMEN}

La discapacidad de tipo motriz puede ser ocasionada por distintos factores y afectar a un número diferente de miembros del cuerpo, siendo la paraplejia la que origina la parálisis completa o parcial de las extremidades inferiores. Las personas que la padecen tienen grandes riesgos en la salud, dada su movilidad limitada, por lo que los ejercicios de rango de movimiento son muy importantes y pueden verse apoyados de ayudas técnicas y tecnologías de apoyo. La presente investigación surge de la necesidad de diseñar un producto que cumpla con los requerimientos de movilidad de miembros inferiores en personas con paraplejia. Dentro de esta primera fase de estudio se realizó una revisión de la literatura, una revisión de patentes, búsqueda de equipos a la venta en el mercado y entrevistas al grupo de estudio y a especialistas en el área, con la finalidad de comprender y especificar el contexto de la investigación.

PALABRAS CLAVE: Paraplejia; discapacidad motriz; ejercicio en paraplejia; terapia física en paraplejia; ayudas técnicas.

\begin{abstract}
Motor disabilities can be caused by different factors and affect different number of body segments, being the paraplegia that causes complete or partial paralysis of the lower extremities. People suffering from paraplegia due to their limited mobility have major health risks, so range of movement exercises are very important, which can be supported by technical aids and assistive technologies. The present investigation arises from the need to design a product that meets the lower limbs mobility requirements in people with paraplegia. Within this first phase of the study, a literature review was carried out, also a patent review, search for equipment for sale in the market and interviews to the study group and specialists in the area, this in order to understand and specify the context of the research.
\end{abstract}

KEYWORDS: Paraplegia; motor disability; exercise in paraplegia; physical therapy in paraplegia; technical assistance.

Correspondencia:

DESTINATARIO: Marlon Miranda Bañuelos INSTITUCIÓN: Universidad Autónoma de Ciudad Juárez DIRECCIÓN: Avenida del Charro 450, Partido Romero, C. P. 32310, Ciudad Juárez, Chihuahua, México. CORREO ELECTRÓNICO: al171777@alumnos.uacj.mx
Fecha de recepción:

31 de julio de 2019

Fecha de aceptación:

4 de septiembre de 2019 


\section{INTRODUCCIÓN}

En México, acorde al Módulo de Condiciones Socioeconómicas de la Encuesta de Ingresos y Gastos de los Hogares del INEGI ${ }^{[1]}$, la población que presenta algún tipo de discapacidad es de aproximadamente $7.7 \mathrm{mi}$ llones de personas y las tres principales discapacidades son la motriz (56.1\%), la visual $(32.7 \%)$ y la auditiva $(18.3 \%)$.

Las discapacidades de tipo motriz pueden ser ocasionadas por distintos factores y afectar a un número diferente de miembros del cuerpo. La mayor cantidad de pacientes con lesiones medulares tiene como resultado una paraplejia (parálisis de la mitad inferior del cuerpo que se debe a una lesión nerviosa en el cerebro o en la médula espinal), siendo un diagnóstico más frecuente que la tetraplejia ${ }^{[2]}$, parálisis causada por una enfermedad o lesión que tiene como consecuencia la pérdida parcial o completa del uso de las cuatro extremidades y del torso. La paraplejia puede tener varios niveles según el daño en la médula espinal, siendo la del nivel T1-T9 en la que se pierde mayormente el control sobre los miembros inferiores del cuerpo, falta de movilidad que ocasiona problemas físicos, psicológicos y socioeconómicos en la persona afectada ${ }^{[3]}$.

Los problemas físicos ocasionados por la paraplejia pueden ser tratados mediante terapia física, la cual se puede apoyar de ayudas técnicas diseñadas para problemas físicos específicos, sin embargo, no todas las personas afectadas tienen acceso a estas terapias y ayudas técnicas, por ejemplo, "en muchos países de ingresos bajos o medianos, solo entre el 5\% y el 15\% de las personas que necesitan dispositivos y tecnologías de asistencia tienen acceso a ellos" ${ }^{[4]}$, situación que se complica aún más, pues hay un vínculo entre la discapacidad física y la pobreza debido a las limitaciones para acceder a una mejor calidad de vida ${ }^{[5]}$.

La práctica del diseño no es una disciplina que se desarrolle independientemente de otras profesiones $y$ actividades, sino que se requiere su relación con otras áreas para llevarla a cabo. Para dar respuesta a las necesidades de las personas, es necesario que el diseñador analice todos los elementos con los que se puede encontrar, por lo tanto, requiere de habilidades que le exigen resolver situaciones complejas en contextos muy variados.
Con base en la necesidad que tienen las personas con paraplejia de movilizar sus miembros inferiores y la asistencia que pueden tener en equipos de apoyo, se ha planteado el diseño de una ayuda técnica que cumpla con las necesidades físicas y socioeconómicas de este grupo de la población. Como metodología para llevar a cabo la investigación, se tomó la teoría de Diseño Centrado en el Usuario, la cual ha sido objeto de estudio de estándares internacionales, actualmente con la norma ISO 9241-210:2010, de la cual se tomaron como base sus cuatro actividades principales. Partiendo de la primera actividad: entendimiento y especificación del contexto de uso ${ }^{[6]}$, se realizó una revisión de la literatura, entrevistas al grupo de estudio y especialistas en el área, y una revisión de patentes. La información presentada en este artículo corresponde a la primera parte de la investigación y posteriormente se realizará el diseño de una ayuda técnica para personas con paraplejia.

\section{REVISIÓN DE LA LITERATURA}

\section{A. Antecedentes}

En el artículo "Rehabilitación de lesiones de la médula espinal" ${ }^{[7]}$ se tratan las causas que pueden ocasionar una lesión de médula espinal, los niveles de lesión, las consecuencias y complicaciones físicas, psicológicas y sociales que trae consigo este tipo de traumas y los niveles de rehabilitación. En este estudio se concluye que las lesiones de la médula espinal conducen a una discapacidad grave. El proceso de tratamiento y rehabilitación es largo, costoso, requiere un enfoque multidisciplinario y cuando se da en forma temprana previene complicaciones.

El proyecto de investigación llamado "Ejercicio para mejorar el flujo sanguíneo y la salud vascular en las extremidades inferiores de parapléjicos" ${ }^{[8]}$ consistió en cuantificar la efectividad de las modalidades de movimiento pasivo de las extremidades inferiores y el ejercicio de la parte superior del cuerpo, para aumentar el flujo sanguíneo en las piernas de los parapléjicos. Esta investigación estuvo basada en la premisa de que después de sufrir una lesión de la médula espinal, un parapléjico sufre una reestructuración vascular drástica y perjudicial que conduce a numerosas consecuencias para la salud. Como conclusión de este estudio, se obtuvo que los episodios repetidos de movimiento pasivo de las extremidades, intercalados con un perio- 
do de recuperación de un minuto, tienen la capacidad de inducir una respuesta hiperémica sostenida, lo cual significa un aumento en la irrigación sanguínea a los miembros inferiores.

En el artículo "La rehabilitación terapéutica a pacientes parapléjicos: impacto desde las tecnologías" [9] se planteó el diseño de un programa de atención integral para el lesionado medular, en el cual se presenta una estructura de investigación con una selección minuciosa de ejercicios de diferentes autores y se menciona que la tecnología posibilita la recuperación de pacientes parapléjicos, apoyándose en un programa de rehabilitación física, sistemático e intensivo, con el que es posible lograr la disminución del proceso de las posteriores afectaciones.

\section{B. Lesiones medulares}

La lesión de médula es una patología estudiada desde la antigüedad, tal como lo demuestran los testimonios encontrados en la cultura egipcia. En los escritos de Hipócrates, en su historia de las deficiencias, sitúa la primera definición de la lesión de médula, y fue descrita como una dolencia que no puede ser tratada en el papiro quirúrgico de Edwin Smith, el texto de carácter médico más antiguo conocido, redactado probablemente en el tercer milenio a. C., copiado en torno a 1600 a. C. y encontrado en 1862 por el egiptólogo americano que le da nombre ${ }^{[10]}$.

Es al finalizar la II Guerra Mundial, con las investigaciones del médico inglés Ludwig Guttmann (19011981), una autoridad en lesionados medulares y fundador del Centro de Lesionados Medulares de Stoke Mandeville, cuando se producen grandes avances en el estudio, el tratamiento y la rehabilitación de la lesión medular. Este autor establece las bases para la concepción de la rehabilitación de los lesionados medulares, lo que permite reconocer la importancia de los factores psicológicos ${ }^{[11]}$.

Se define a las lesiones de la médula espinal como el daño que conlleva déficit neurológico con efectos a largo plazo que persisten toda la vida. Estas alteraciones habitualmente se presentan por debajo del nivel de la lesión ${ }^{[3]}$.

Existen varias formas de clasificar la lesión medular: según su causa, en traumática y no traumática; de acuerdo con el mecanismo de lesión, en lesión por hiperflexión, por flexión con rotación, por hiperextensión y por compresión; según su nivel de lesión en cervical, dorsal y lumbosacra; y de acuerdo con la extensión, en completa e incompleta ${ }^{[3]}$.

\section{Paraplejia}

En Hoppenfeld ${ }^{[12]}$, a la parálisis completa o parcial de las extremidades inferiores y/o porción inferior del cuerpo se le denomina paraplejia. Es causada principalmente por lesión traumática de la columna vertebral (lesión medular), pero también puede deberse a varias enfermedades, tales como mielitis transversa, lesiones quísticas de la médula y paraplejia de Pott (producida por tuberculosis), así como otra serie de trastornos.

Los cambios en el cuerpo son difíciles de afrontar para las personas con paraplejia debido a que de un momento a otro se enfrentan a no volver a caminar, no tener sensibilidad superficial ni profunda por debajo del nivel de la lesión y el riesgo de complicaciones como depresión, infecciones urinarias, úlceras de presión, estreñimiento e impactación fecal, dolor neuropático, espasticidad y problemas osteoarticulares ${ }^{[13]}$.

El paciente parapléjico en más de un $80 \%$ de los casos sufre de lesiones irreversibles que impedirían en el futuro un restablecimiento de la movilidad de sus miembros inferiores y necesitará un desarrollo muscular lo suficientemente potente para brazos y tronco que supla la inactividad de los miembros inferiores ${ }^{[2]}$.

La alteración de la movilidad y la sensibilidad implican la utilización permanente de toda clase de equipos y aditamentos, afecta la habilidad para realizar las actividades de la vida diaria e incrementa la dependencia y el riesgo de complicaciones como depresión, infecciones urinarias, úlceras de presión, estreñimiento e impactación fecal, dolor neuropático, espasticidad y problemas osteoarticulares ${ }^{[13]}$.

La reducción en la salud cardiovascular observada en personas con una lesión de médula espinal imita lo que se observa en el proceso de envejecimiento normal, pero a un ritmo mucho más rápido. Una combinación mortal de tasa metabólica reducida, disminución de la masa muscular, aumento de la masa grasa y opciones de ejercicio limitadas aumentan el riesgo de desarrollar enfermedad cardiovascular. Además, las personas con 
lesión de médula espinal comúnmente tienen tasas elevadas de dislipidemia (niveles excesivamente elevados de colesterol o grasas en la sangre) e hiperinsulinemia (cantidad de insulina en la sangre es mayor que la que se considera normal) en comparación con la población sana ${ }^{[8]}$.

Después de la incursión de una lesión de médula espinal, hay un cambio severo y rápido en la fisiología de todo el cuerpo, especialmente por debajo del nivel de la lesión. Algunas complicaciones médicas comunes experimentadas en esta población son la atrofia muscular y los trastornos del metabolismo óseo. Se ha establecido que los periodos crónicos de descarga muscular pueden dar como resultado una reducción severa en el volumen muscular debido a la disminución de las áreas transversales musculares. La atrofia muscular representa el deterioro del cuerpo por la ausencia de estimulación del sistema nervioso, reduciendo el tono muscular ${ }^{[13]}$.

Cualquier población en la que la movilidad es limitada, tiene mayores riesgos de formación de úlceras por presión debido a las tasas reducidas de flujo sanguíneo y la acumulación de sangre en las extremidades. Las personas con una lesión de médula espinal completa tienen un riesgo elevado de formación de úlceras por presión en todo momento después de la lesión ${ }^{[8]}$.

Los profesionales que han trabajado con individuos con lesiones espinales saben que los ejercicios pasivos de rangos de movimiento son importantes, las articulaciones inmóviles sufren cambios químicos, los tejidos se contraen, se tensionan y se recogen. Estos ejercicios no funcionan con articulaciones contraídas y una vez que esto pasa, solo puede ser corregido con cirugía. Pero se pueden hacer dos cosas para evitar contracturas y dolor: las articulaciones deben obtener a diario movimiento pasivo en todo su rango, deben ser estiradas con una tensión modesta y se debe mantener esta posición por unos momentos, si es necesario ${ }^{[14]}$.

Los programas de movimientos activos (realizados por el paciente con su propia fuerza de forma voluntaria o autorrefleja y controlada, corregidos o ayudados) y pasivos (se aplican sobre las estructuras afectadas, sin que el paciente realice ningún movimiento voluntario de la zona que hay que tratar), comienzan tan pronto sea posible después de la lesión para evitar la rigidez en los miembros y articulaciones afectadas; la limitación de ellos podría condicionar la capacidad de realizar tareas básicas en el futuro ${ }^{[15]}$.

Actualmente, una de las modalidades comunes de ejercicio-terapia usada en lesiones de médula espinal incluyen el ejercicio de la parte superior del cuerpo (por ejemplo: ergómetros de brazos, ergómetros de sillas de ruedas, cintas de correr para sillas de ruedas, libre movimiento de brazos, aeróbicos sentados, natación y deportes en silla de ruedas) ${ }^{[8]}$.

El ejercicio de la parte superior del cuerpo, cuando se usa en combinación con el movimiento pasivo de las extremidades inferiores, tiene la capacidad de invocar un gran aumento en el flujo sanguíneo de la arteria femoral, lo cual podría tener una aplicación clínica importante para esta población ${ }^{[8]}$.

Con un protocolo de movimiento pasivo de miembros inferiores durante el ejercicio de la parte superior del cuerpo, con el estímulo fisiológico combinado de ambas modalidades, el aumento del gasto cardíaco durante el ejercicio de la parte superior del cuerpo y la vasodilatación durante el movimiento pasivo de las extremidades inferiores, puede provocar una respuesta hiperémica (aumento en la irrigación sanguínea a un órgano o tejido) mayor en comparación a cuando estas modalidades se utilizan de forma aislada ${ }^{[8]}$.

De acuerdo con la literatura, en personas con lesión de médula espinal, el movimiento pasivo de miembros induce a un aumento significativo, aunque transitorio, en la irrigación sanguínea en la arteria femoral. Los estudios de Venturelli y sus colegas ${ }^{[16]}$ mostraron cómo el flujo sanguíneo de la arteria femoral casi duplicó los valores iniciales durante el movimiento pasivo de la pierna de una persona paralítica, esto a través de un rango de movimiento de 0 a $90^{\circ}$ de flexión ${ }^{[8]}$.

Los ejercicios pasivos, activos asistidos, activos y de resistencia, así como el ciclismo y los ejercicios acuáticos, reducen la atrofia muscular, las úlceras de presión, la inactividad, obesidad y fracturas óseas en el paciente parapléjico, y deben ser compatibles con el nivel de lesión. Los ejercicios pasivos de rango de movimiento deben hacerse al menos una vez al día y en presencia de espasticidad al menos 2-3 veces al día ${ }^{[7]}$.

La movilización pasiva, activa y activa asistida de las articulaciones es esencial, dado que permite evitar las 
contracturas, el dolor y la limitación funcional de las articulaciones afectadas, así como prevenir atrofias y debilidad en la musculatura ilesa ${ }^{[2]}$.

Además de reducir el riesgo de enfermedad cardíaca, la investigación en la población con lesión de médula espinal ha demostrado que el ejercicio mejora la respiración, la fuerza muscular, la circulación, la composición corporal, la autoestima, la autoconfianza, la depresión, la ansiedad y la independencia. También ayuda a prevenir complicaciones secundarias, como infecciones del tracto urinario, úlceras por presión e infecciones respiratorias; reduce el riesgo de diabetes; mejora la función del sistema inmunitario y disminuye el estreñimiento ${ }^{[17]}$.

Las ayudas técnicas existentes son auxiliares en las deficiencias de las personas con discapacidad física, tratando de acercarlas lo más posible a la normalidad. De acuerdo con la definición de la Organización Internacional de Normalización, se llama ayudas técnicas a todos aquellos productos, instrumentos, equipos o sistemas técnicos utilizados por una persona con discapacidad, fabricados especialmente o disponibles en el mercado, para prevenir, compensar, mitigar o neutralizar una deficiencia, discapacidad o minusvalía ${ }^{[18]}$.

El equipo y ayudas técnicas para compensar la falta de movilidad de miembros inferiores pueden utilizarse principalmente en centros de terapia física y rehabilitación, sin embargo, hay barreras que interfieren con compromisos a largo plazo para personas con lesión de médula espinal. Estos impedimentos pueden incluir falta de acceso a la instalación de ejercicio, carencia de transporte público accesible, etc. ${ }^{[19]}$.

Las personas con algún tipo de discapacidad presentan grandes necesidades. Según Urteaga ${ }^{[18]}$, "es un hecho a tener en cuenta que muchísimas de estas personas necesitan ayuda a la hora de realizar las actividades de la vida diaria. Bien ayuda personal o de ayudas técnicas. De la misma manera que se refiere que muchas personas creen necesitar algún tipo de ayuda y no la reciben".

El ejercicio después de una lesión de médula espinal puede ser tanto un objetivo hacia el estado físico (cardiovascular o muscular), como compensatorio (con dispositivo de asistencia) y restaurativo (estimulación eléctrica funcional y entrenamiento locomotor) ${ }^{[8]}$.
Con un programa de rehabilitación física organizado se pueden disminuir las afectaciones futuras ${ }^{[2]}$.

De acuerdo con Urteaga y Aguinaga ${ }^{[18]}$, los distintos tipos de ayudas técnicas y tecnologías desarrollados para las personas que sufren de discapacidad, permiten mejorar su calidad de vida, desarrollando habilidades para vivir de forma más autónoma y aminorando los efectos secundarios de la falta de movimiento de los miembros del cuerpo afectados.

Acorde a la información revisada sobre paraplejia y el impacto que esta tiene en las personas que la padecen, se visualiza como de suma importancia para esta población el desarrollo de ayudas técnicas económicamente accesibles que promuevan el movimiento pasivo de las extremidades inferiores.

\section{El diseñador como factor de cambio}

Es posible identificar al diseñador como un factor de cambio en la sociedad, pues su trabajo da respuesta a las necesidades de las personas, lo cual otorga a su labor una responsabilidad social importante, como menciona Rispoli ${ }^{[20]}$. La idea de "trabajar para un mundo mejor" acompaña al diseño ya a partir de su nacimiento, sin embargo, de acuerdo a este mismo autor, desde 1970 es posible detectar un verdadero momento de inflexión en lo que a conciencia social y ambiental del diseñador se refiere.

En base al objetivo de la investigación de diseñar un producto que cumpla con las necesidades de movilización de miembros inferiores en personas con paraplejia y la necesidad de una metodología para llevar a cabo la investigación, se decidió utilizar la teoría de Diseño Centrado en el Usuario, por su enfoque en las necesidades e intereses del usuario, y el especial hincapié en hacer que los productos sean utilizables y comprensibles ${ }^{[21]}$.

El diseño centrado en el usuario ha sido objeto de estudio de estándares internacionales, principalmente el ISO 9241-210, el cual reemplaza al ISO 13407:1999. Esta norma internacional define cuatro actividades principales a realizarse:

a) entender y especificar el contexto de uso, b) especificar los requerimientos del usuario, 
c) producir soluciones de diseño y

d) evaluar el diseño.

Estas actividades son llevadas a cabo de forma iterativa en todo el proceso de diseño hasta que la solución cumple con los requisitos del usuario ${ }^{[6]}$.

\section{E. Patentes y productos existentes en el mercado}

Se realizó una búsqueda de patentes y artículos existentes en el mercado que pudieran tener similitudes con el diseño de la ayuda técnica que se pretende diseñar en esta investigación. Del total de patentes encontradas, la Tabla 1 presenta las siete que comparten más similitudes con el planteamiento de la hipótesis de la investiga- ción: el diseño de una ayuda técnica para movilizar los miembros inferiores de personas con paraplejia puede mejorar su salud física.

Los diseños de estas patentes tienen en común que funcionan con un mecanismo mecánico y que movilizan las piernas del usuario usando la fuerza de los brazos, y algunas de estas tienen la opción de trabajar miembros superiores e inferiores de manera aislada. En la Tabla 2 se muestran los resultados de la búsqueda de este tipo de productos a la venta en el mercado que en la mayoría de los casos tienen un costo elevado, comparten la característica de funcionar con un mecanismo eléctrico y están enfocados a la terapia física y rehabilitación, con los cuales se trabajan miembros inferiores y superiores.

TABLA 1.

Patentes de Productos Encontrados

\begin{tabular}{|c|c|c|}
\hline Patente y utilización & DESCRIPCIÓN Y TIPO DE MECANISMO & Dibujo \\
\hline $\begin{array}{l}\text { Dual action recumbent exercise cycle (Número } \\
\text { de patente US 8,113,996 B1). } \\
\text { Aparato de ejercicios / terapia física. }\end{array}$ & $\begin{array}{l}\text { Aparato dirigido a las necesidades de personas } \\
\text { con obesidad, así como con problemas de } \\
\text { equilibrio como la enfermedad de Parkinson, } \\
\text { esclerosis múltiple y accidente cerebrovascular. } \\
\text { Mecanismo mecánico. }\end{array}$ & \\
\hline $\begin{array}{l}\text { Aparato de ejercicios sentado US2009075786A.) } \\
\text { Aparato de ejercicios. }\end{array}$ & $\begin{array}{l}\text { Aparato para ejercitar. } \\
\text { Mecanismo mecánico. }\end{array}$ & \\
\hline $\begin{array}{l}\text { Recumbent leg and arm stepping exercising } \\
\text { apparatus US5505679A. } \\
\text { Aparato de ejercicios. }\end{array}$ & $\begin{array}{l}\text { Se puede usar como remo, stepper y/o realizar } \\
\text { ejercicio tipo bench press. } \\
\text { Mecanismo mecánico. }\end{array}$ & \\
\hline $\begin{array}{l}\text { Exercise apparatus } \\
\text { US2012077653A. } \\
\text { Aparato de ejercicios. }\end{array}$ & $\begin{array}{l}\text { Aparato de ejercicio que incluye una guía y } \\
\text { un miembro deslizante montados de forma } \\
\text { deslizante en la guía. } \\
\text { Mecanismo mecánico. }\end{array}$ & \\
\hline $\begin{array}{l}\text { Recumbent stepper apparatus } \\
\text { US2009124467A. } \\
\text { Aparato de terapia física y ejercicios. }\end{array}$ & $\begin{array}{l}\text { Un dispositivo de ejercicio reclinado que } \\
\text { proporciona acondicionamiento cardiovascular } \\
\text { para parte inferior y superior del cuerpo. } \\
\text { Mecanismo mecánico. }\end{array}$ & \\
\hline $\begin{array}{l}\text { Recumbent stepping exercise device with } \\
\text { stimulation and related methods US7996080B. } \\
\text { Aparato de ejercicios. }\end{array}$ & $\begin{array}{l}\text { Facilita la realización del ejercicio utilizando la } \\
\text { estimulación. } \\
\text { Mecanismo mecánico. }\end{array}$ & \\
\hline $\begin{array}{l}\text { Recumbent stepper exercise machine } \\
\text { US7713176B. } \\
\text { Aparato de ejercicios. }\end{array}$ & $\begin{array}{l}\text { Una máquina reclinada para hacer ejercicio, que } \\
\text { comprende: un bastidor; un asiento apoyado } \\
\text { desde dicho bastidor; un mecanismo de } \\
\text { accionamiento soportado en dicho marco. } \\
\text { Mecanismo mecánico. }\end{array}$ & \\
\hline
\end{tabular}


TABLA 2.

Productos Encontrados en el Mercado

\begin{tabular}{|c|c|c|}
\hline Producto y Utilización & DESCRIPCIÓN, PRECIO Y SITIO WEB DE VENTA & IMAGEN \\
\hline $\begin{array}{l}\text { Konliking } 180 \mathrm{w} \text { Terapia Física Electrónica Y } \\
\text { Rehab Bike. } \\
\text { Terapia física y rehabilitación. }\end{array}$ & $\begin{array}{l}\text { Mecanismo de tipo eléctrico. } \\
\$ 65,620 \text { pesos } \\
\text { mercadolibre.com }\end{array}$ & \\
\hline $\begin{array}{l}\text { Bicicleta de Fisioterapia } \\
\text { Terapia física y rehabilitación. }\end{array}$ & $\begin{array}{l}\text { Mecanismo de tipo eléctrico. } \\
€ 735,00 \text { euros } \\
\text { aliexpress.com }\end{array}$ & \\
\hline $\begin{array}{l}\text { Bicicleta de terapia física/ rehabilitación brazo } \\
\text { y pierna. } \\
\text { Terapia física y rehabilitación. }\end{array}$ & $\begin{array}{l}\text { Mecanismo de tipo eléctrico. } \\
\$ 1,790 \text { dólares USD } \\
\text { amazon.com }\end{array}$ & \\
\hline $\begin{array}{l}\text { MS350 Recumbent Stepper with removable seat } \\
\text { for Wheelchair access } 735015 \text {. } \\
\text { Terapia física y rehabilitación. }\end{array}$ & $\begin{array}{l}\text { Mecanismo de tipo eléctrico. } \\
\$ 5,399.00 \text { dólares USD } \\
\text { goldmedicalsupplies.com }\end{array}$ & \\
\hline $\begin{array}{l}\text { SciFit StepONE Recumbent Stepper 10-6090. } \\
\text { Terapia física y rehabilitación. }\end{array}$ & $\begin{array}{l}\text { Mecanismo de tipo eléctrico. } \\
\$ 4,885.71 \text { dólares USD } \\
\text { goldmedicalsupplies.com }\end{array}$ & \\
\hline $\begin{array}{l}\text { PhysioStep PRO. } \\
\text { Terapia física y rehabilitación. }\end{array}$ & $\begin{array}{l}\text { Mecanismo de tipo eléctrico. } \\
\$ 4,699 \text { dólares USD } \\
\text { hcifitness.com }\end{array}$ & \\
\hline
\end{tabular}

\section{F. Resultados de entrevistas a pacientes y profesionales}

Se realizaron 14 entrevistas al grupo de estudio, en el que participaron 8 mujeres y 6 hombres, con un rango de edad en su mayoría de entre 45 a 59 años de edad. Los diagnósticos médicos más recurrentes fueron lesión medular, neuropatía diabética y paraplejia. De los entrevistados, 7 realizaban terapia física y rehabilitación, mediante las técnicas de reforzamiento de piernas, terapia motriz, fortalecimiento de brazos, estas con apoyo en hidroterapia, mecanoterapia y electroterapia.

De las personas que indicaron realizar terapia física, todas reportaron resultados positivos, 6 personas reportaron apoyarse en algún tipo de dispositivo para realizar la terapia física (Figura 1), 13 de los partici- pantes consideraron necesarios nuevos productos enfocados a terapia física y rehabilitación de problemas motrices (Figura 2)

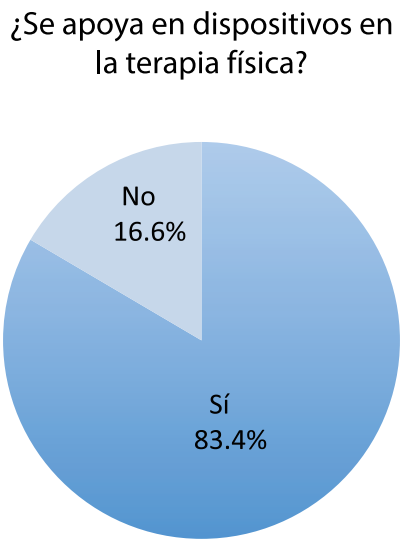

Figura 1. Apoyo en dispositivos para realizar terapia física. 


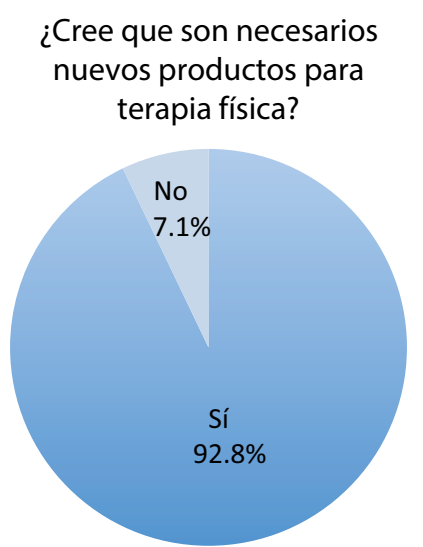

Figura 2. Necesidad de nuevos productos.

Las entrevistas a especialistas en terapia física y rehabilitación se aplicaron a 18 expertos (16 fisioterapeutas y 2 médicos especialistas en rehabilitación), y los resultados obtenidos de estas fueron los siguientes: las actividades más recurrentes llevadas a cabo en pacientes parapléjicos fueron activación de los músculos inactivos por medio de electroestimulación, movilizaciones activas y pasivas de miembros inferiores, ejercicios de fortalecimiento y aplicación de calor (Figura 3).

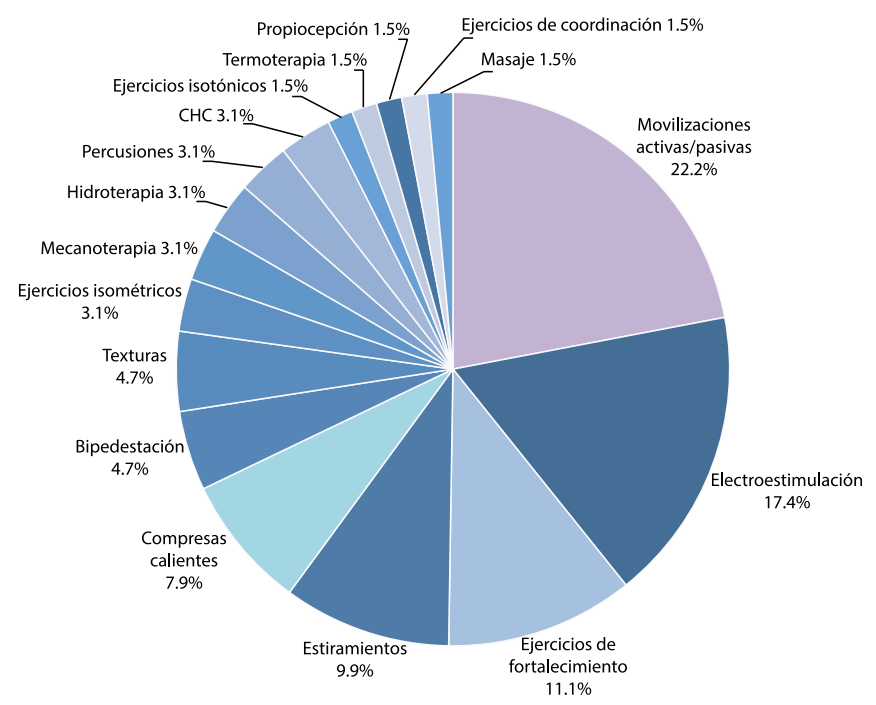

Figura 3. Actividades más recurrentes.

El equipo y ayudas técnicas más utilizados fueron equipo de electroestimulación, equipo de bipedestación, férulas, compresas y mecanoterapia, que son auxiliares en el fortalecimiento de músculos, para ayudar en el movimiento, la activación muscular, el tono muscular, la estimulación del sistema nervioso, la disminución de la espasticidad, y contribuyen en la eliminación de contracturas y en evitar úlceras de presión (Figura 4).

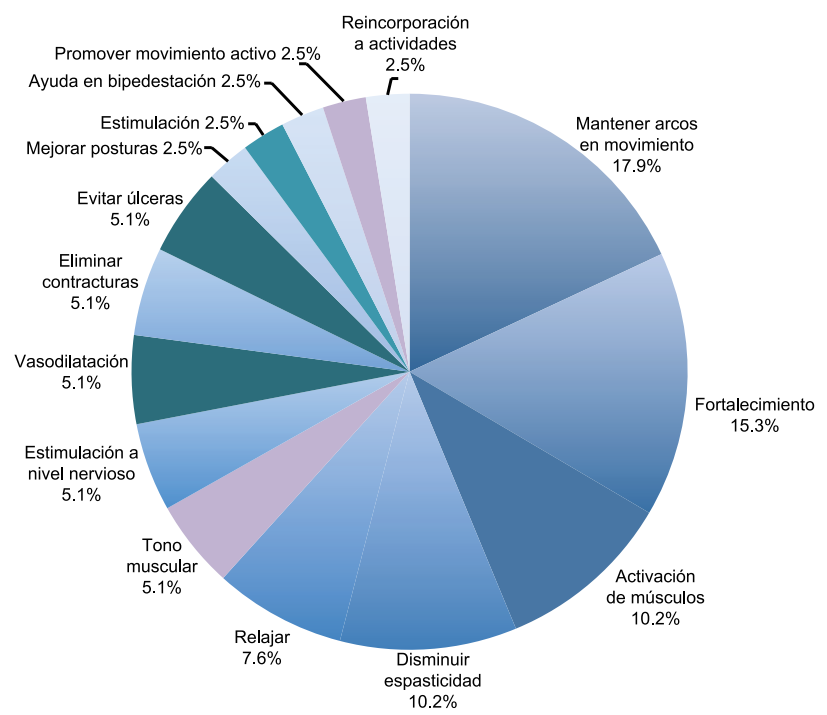

Figura 4. Principales contribuciones del equipo utilizado en las terapias físicas.

Los especialistas recomendaron realizar terapia física en el hogar para una más pronta rehabilitación, y comentaron que sería ideal para el paciente contar con equipo de apoyo. Sobre las ayudas técnicas disponibles en el mercado consideraron que no son fáciles de adquirir por su alto costo por lo que en general se encuentran principalmente en centros de rehabilitación, además de que estimaron que por sus proporciones no son prácticas para tener en el hogar.

Las respuestas de los especialistas en cuanto al diseño de un nuevo producto que cumpla con la movilización de miembros inferiores, fueron que el paciente tendría que ser candidato para poder utilizarlo, su uso debería ser bajo supervisión y sería de gran utilidad para la salud y debería poder ser empleado por personas de cualquier edad. Las recomendaciones para el producto fueron: la utilización de metales para la estructura y materiales blandos para otras partes como el asiento; que los materiales empleados sean resistentes, ligeros y sin filos o bordes rectos; que el producto no ocupe mucho espacio, tenga un costo accesible, sea ligero, ergonómico, de fácil traslado, fácil de usar, diseño antiderrapante, con sujetadores para pies, bandas de seguridad, separador de muslos y como medida de seguridad el uso de guardas para mecanismos.

\section{G. Revisión de artículos relacionados}

Se hizo una búsqueda de artículos relacionados a esta revisión de la literatura, las fuentes consultadas fue- 
ron las bases de datos Scielo, Dialnet, PubMed, DOAJ, Science Direct y PEDro. La búsqueda se limitó a los idioma español e inglés y se utilizaron las siguientes palabras clave: discapacidad motriz, motor disability, paraplejia, paraplegia, spinal cord injury, lesiones medulares, revisión de literatura, literature review, review of the literature, review article, artículo de revisión, diseño, design, assistive technologies y ayudas técnicas.

Como resultado de la búsqueda se encontraron solamente cuatro documentos con una relación más directa a este artículo de revisión: dos en Dialnet, uno en Scielo y otro en PubMed.

En el artículo "Lesión medular y ejercicio físico: revisión desde una perspectiva deportiva" ${ }^{[22]}$ se llevó a cabo un análisis de las alteraciones físicas provocadas por las lesiones medulares, con la finalidad de aportar información sobre estas y su interacción con la práctica deportiva, y en las conclusiones se destacan los beneficios de la práctica habitual del ejercicio físico en personas con lesiones medulares.

En el documento "Assistive technologies for self-managed pressure ulcer prevention in spinal cord injury: A scoping review" ${ }^{[23]}$ se trata un problema persistente en personas con lesiones de médula espinal: las úlceras de presión. En este estudio se analizan tecnologías de autocontrol para la prevención de úlceras, se hizo una clasificación de estas tecnologías en categorías, las cuales se identificaron en diferentes estudios durante la revisión y se tuvo como conclusión que estas tecnologías de autogestión tienen una efectividad de baja a moderada, por lo que se hizo la recomendación de desarrollar tecnologías integradas que aborden múltiples factores de riesgo.

En el artículo "Revisión sobre aspectos genéricos acerca de la actividad física adaptada en la persona con lesión medular" ${ }^{[24]}$ se hizo una búsqueda en varias bases de datos de artículos que destacaran la importancia de la actividad física adaptada en personas con lesión medular. En esta revisión se tuvo como conclusión que se deben seguir promoviendo políticas que hagan accesible la actividad física adaptada a la población con discapacidad, ya que se destaca un aumento en la calidad de vida.

El artículo "Lesiones medulares y discapacidad: revisión bibliográfica" ${ }^{[25]}$ hizo una revisión considerando una estimación integral, tomando en consideración no solo la salud, sino también los aspectos psicológico y social, llegando a la conclusión de que las políticas, programas y acciones deben ser mas integradoras para lograr la mayor independencia y autonomía posibles.

\section{CONCLUSIONES}

Los programas de movimiento de miembros inferiores son indispensables para la salud de las personas con paraplejia, ya que la actividad física ayuda a reducir los efectos secundarios ocasionados por la falta de movilidad.

Con base en la revisión de literatura, de patentes y las entrevistas realizadas, se ha determinado que es necesario un producto que cumpla con las necesidades físicas y socioeconómicas de las personas con paraplejia, por lo tanto, con la información obtenida se hizo un listado con las necesidades a tomar en cuenta para el diseño del producto, quedando en esta solo aquellas que estuvieran lo suficientemente fundamentadas, esto mediante un consenso entre el investigador y los asesores del proyecto (Figura 5).

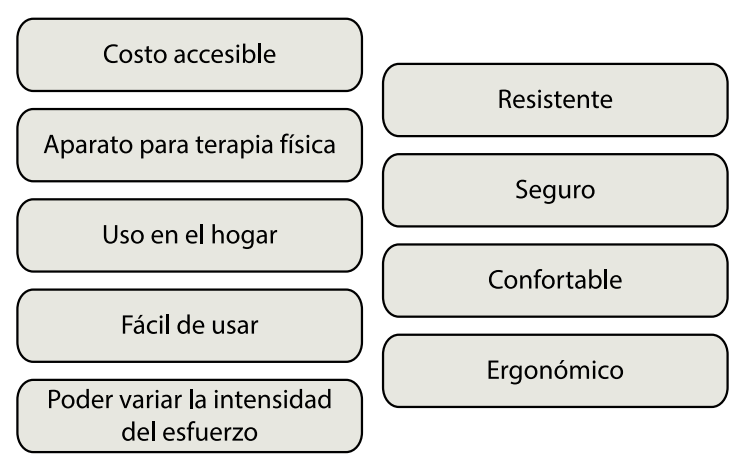

Figura 5. Necesidades de diseño obtenidas en base al análisis de la información.

El producto diseñado debe permitir al usuario realizar el movimiento de miembros inferiores de forma ergonómica, segura y funcional, este debe ser entendible y fácil de usar, además de que debe contar con las características necesarias para su uso en el hogar y ser accesible económicamente.

La presente revisión de literatura y las revisiones encontradas en diferentes bases de datos son similares en que se resalta la necesidad de la actividad física en personas con lesión de médula espinal, pero solo en uno de esos artículos, "Assistive technologies for self-mana- 
ged pressure ulcer prevention in spinal cord injury: A scoping review" [23], se abordan tecnologías de asistencia, sin embargo no se trata de ayudas técnicas sino de softwares y programas de entrenamiento. La principal diferencia entre esta revisión y los documentos encontrados es el objetivo: mientras que este estudio tuvo como finalidad el diseño de una ayuda técnica, ninguna de las revisiones encontradas tuvo esa finalidad.

\section{REFERENCIAS}

[1] INEGI, Módulo de Condiciones Socioeconómicas de la Encuesta de Ingresos y Gastos de los Hogares del INEGI. México: INEGI, 2014.

[2] Á. Hidalgo-Martínez, "La rehabilitación terapéutica a pacientes parapléjicos: impacto desde las tecnologías", Podium, vol. 12. no. 1, pp. 21-30, enero-marzo 2017.

[3] K. Strassburguer-Lona, S. Hernández-Porras, E. Barquín-Santos, Lesión Medular: Guía para manejo integral del paciente con LM crónica. Madrid: Aspaym, 2014, pp. 1-161.

[4] OMS, Informe Mundial sobre la Discapacidad. Ginebra: OMS, 2011.

[5] M. R. Rocha-Rodríguez, M. Cruz-Ortiz, M. C. Pérez-Rodríguez, J. G. Mendoza-Zapata, "Pobreza y discapacidad, un vínculo para estudiar a fondo", Waxapa, año 6, no. 10, pp. 18-25, enero-junio 2014. Disponible en: http://www.uan.edu.mx/d/a/publicaciones/revista_waxapa/pdf/waxapa_10.pdf

[6] ISO, ISO 9241-210, 2010, p. 40.

[7] K. Nas, L. Yazmalar, V. Şah, A. Aydın, K. Öneş, "Rehabilitation of spinal cord injuries", World Journal of Orthopedics, vol. 6, no. 1, enero 2015. [En línea]. Disponible en: http://www.wjgnet.com/2218-5836/full/v6/ i1/8.htm

[8] K. J. Burns, "Exercise to improve blood flow and vascular health in the lower limbs of paraplegics", Disertación de doctorado, College of Education, Health, and Human Services, Kent State University, 2015. [En línea]. Disponible en: https://etd.ohiolink.edu/!etd. send_file? accession=kent 1435874538 \&disposition=inline
[9] Á. Hidalgo-Martínez, "La rehabilitación terapéutica a pacientes parapléjicos : impacto desde las tecnologías", Podium, vol. 12, no. 1, pp. 21-30, 2017. 2017. [En línea]. Disponible en: http://podium.upr.edu.cu/index. php/podium/article/view/687

[10] R. Ramos-Machuca, "Las complicaciones secundarias del parapléjico", Tesis de Especialidad (especialista de primer grado de medicina Física y Rehabilitación), Facultad de Ciencias Médicas, La Habana, 1981.

[11] R. González Mas, Rehabilitación Médica. Barcelona, España: Editorial Masson, 1997.

[12] S. Hoppenfeld, Neurología Ortopédica. Ed. El Manual Moderno, 1981.

[13] M. E. Moreno-Fergusson y M. C. P. Amaya-Rey, "Cuerpo y corporalidad en la paraplejia: significado de los cambios", Avances en Enfermería, vol. 30, no. 1, pp. 82 94, 2012

[14] R. Gutierrez, S. Gardner y J. Steward, Problemas de las Articulaciones, Condiciones Secundarias Prevención y Tratamiento, Serie A, no. 5, Kansas: Universidad de Kansas, 1993. [En línea]. Disponible en: https://rtcil. drupal.ku.edu/sites/rtcil.drupal.ku.edu/files/images/ galleries/SCI Joint Problems Spanish.pdf

[15] B. Costa-Morales y R. M. Velasquez, "Programa rehabilitación físico muscular en parapléjicos espasticos de lesión lumbar en 'Asociación para el Fortalecimiento Integral de las Personas con Discapacidad' en Santa Cruz de la Sierra", Universidad, Ciencia y Sociedad, no. 4, pp. 33-41, 2011

[16] M. Venturelli, M. Amann, G. Layec, J. McDaniel, J. D. Trinity, A. S. Fjeldstad, S. J. Yves, G. Yonnet, R. S. Richardson, "Passive leg movement-induced hyperaemia with a spinal cord lesion: evidence of preserved vascular function", Acta Physiol., vol. 210, no. 2, 2014. https:// doi.org/10.1111/apha.12173

[17] K. Kaupang, Get Moving: Exercise and SCI, Northwest Regional Spinal Cord Injury System, febrero 2013. [En línea]. Disponible en: http://sci.washington.edu/info/ forums/reports/exercise_2013.asp

[18] G. Urteaga-Ceberio, Programa de promoción, información, asesoramiento y entrenamiento de las ayudas téc- 
nicas para personas con discapacidad física, sus familiares y profesionales de Navarra, academica-e, 2013. [En línea]. Disponible en: http://academica-e.unavarra.es/ handle/2454/7812

[19] A. S. Gorgey, "Exercise awareness and barriers after spinal cord injury", World J Orthop, vol. 5, no. 3, pp. 158-162, julio 2014. [En línea]. Disponible en: http:// www.wjgnet.com/2218-5836/full/v5/i3/158.htm doi: 10.5312/wjo.v5.i3.158

[20] E. R. Rispoli, "El diseñador como productor. Reflexiones en torno a la idea de responsabilidad social en el diseño contemporáneo", Obra Digital, no. 9, pp.28-41, septiembre 2015. doi: 10.25029/od.2015.65.9

[21] D. A. Norman, La psicología de los objetos cotidianos. Madrid: Nerea, 1990.
[22] G. Brizuela, J. L. Romero y J. Beltrán, “Lesión medular y ejercicio físico: revisión desde una perspectiva deportiva”, Revista Española de Discapacidad, vol. 4, no. 2, 2016.

[23] J. Y. Tung, B. Stead, W. Mann, B. Pham y M. R. Popovic, "Assistive technologies for self-managed pressure ulcer prevention in spinal cord injury: A scoping review", J Rehabil Res Dev, vol. 52, no. 2, 2015. doi: 10.1682/ JRRD.2014.02.0064

[24] M. Á. Capó-juan M. Bennasar-Veny, A. Aguiló-Pons, J. E. de Pedro-Gómez, "Revisión sobre aspectos genéricos acerca de la actividad física adaptada en la persona con lesión medular", Arch Med Deporte, vol. 34, no. 2, pp. 100-104, 2017.

[25] C. P. Henao-Lema y J. E. Pérez-Parra, "Lesiones medulares y discapacidad : revisión bibliográfica”, Aquichan, vol. 10, no. 2, pp. 157-172, agosto 2010. 